\title{
Memoria de trabajo y su relación con el rendimiento académico en estudiantes de medicina
}

\section{Working memory and its relationship with acaemic performance in medicine students}

\author{
VARELA-CERVANTES, Luis Daniel †, RÍOS-VALLES, José Alejandro*, BARRAGÁN-LEDESMA, \\ Laura Ernestina y ESTRADA-MARTINEZ, Sergio
}

Centro de Rehabilitación y Educación Especial (CREE, Durango), Predio Canoas, ISSSTE, Predio Canoas, 34079 Durango, Dgo.

ID $1^{\mathrm{er}}$ Autor: Luis Daniel, Varela-Cervantes / ORC ID: 0000-0001-7419-6083, Researcher ID Thomson: X-3233-2018, CVU CONACYT ID: 953404

ID $1^{\mathrm{er}}$ Coautor: José Alejandro, Ríos-Valles / ORC ID: 0000-0002-8407-3017, Researcher ID Thomson: X-3209-2018, CVU CONACYT ID: 313266

ID $2^{\text {do }}$ Coautor: Laura Ernestina, Barragán-Ledesma / ORC ID: 0000-0001-5929-1648, Researcher ID Thomson: X-92392018, CVU CONACYT ID: 204908

ID $3^{\text {er }}$ Coautor: Sergio, Estrada-Martinez / ORC ID: 0000-0002-7633-6841, Researcher ID Thomson: T-1487-2018, CVU CONACYT ID: 393364

DOI: $10.35429 / \mathrm{JOHS} .2019 .19 .6 .7 .20$

Recibido Abril 10, 2019; Aceptado Junio 25, 2019

\section{Resumen}

Entender qué es la Memoria de Trabajo, cómo funciona y cómo está relacionada con el aprendizaje es muy importante para la educación. Objetivos: Determinar la relación entre la memoria de trabajo con el rendimiento académico de los estudiantes de Medicina Metodología: Se realizó un estudio transversalobservacional, de tipo descriptivo correlacional. El diseño es retrospectivo-retrolectivo con una muestra no probabilística por conveniencia con previa firma de consentimiento informado con un total de 270 estudiantes de la facultad de Medicina de la UJED. Contribución: En los resultados obtenidos no se encontró alguna correlación significativa entre la memoria de trabajo y rendimiento académico, no obstante, se observó un porcentaje alto $(53.3 \%)$ de estudiantes que han recursado al menos una materia y estos cuentan con mejores calificaciones que el grupo que no ha recursado materias, por ello se realizó un segundo análisis mediante una prueba comparativa con U de MannWithney, encontrando una diferencia significativa entre las calificaciones de los grupos con una $\mathrm{p}=.000$, no así para los componentes de la memoria de trabajo los cuales se encontraron dentro de valores normales según la prueba neuropsicológica breve en español.

Memoria de trabajo, Rendimiento Académico, Estudiantes de Medicina

\begin{abstract}
Understanding what the Working Memory is, how it works and how it is related to learning is very important for education. Objectives: To determine the relationship between working memory and the academic performance of medical students. Methodology: A cross-observational study, of a descriptive correlational type, was carried out. The design is retrospectiveretrolective with a non-probabilistic sample for convenience with prior signature of informed consent with a total of 270 students of the UJED School of Medicine. Contribution: In the results obtained, no significant correlation was found between working memory and academic performance, however, a high percentage $(53.3 \%)$ of students who have attended at least one subject were observed and they have better grades than the group that has not studied subjects, for that reason a second analysis was carried out by means of comparative test with U Mann-Withney, finding a significant difference between the qualifications of the groups with a $\mathrm{p}=.000$, not so far the memory components of working memory which were found within normal values according to the brief neuropsychological test in Spanish.
\end{abstract}

Working Memory, Academic Performance, Medicine Students

Citación: VARELA-CERVANTES, Luis Daniel, RÍOS-VALLES, José Alejandro, BARRAGÁN-LEDESMA, Laura Ernestina y ESTRADA-MARTINEZ, Sergio. Memoria de trabajo y su relación con el rendimiento académico en estudiantes de medicina. Revista de Ciencias de la Salud. 2019. 6-19: 7-20.

*Correspondencia al Autor (Correo Electrónico: alexriva@ hotmail.com)

$\dagger$ Investigador contribuyendo como primer Autor 


\section{Introducción}

En el proceso de educación se presentan dificultades en el aprendizaje de conocimientos que se manifiestan en bajas calificaciones, reprobación de materias, deserción, tiempo extra de estancia en la universidad y dificultad para concluir los requisitos para lo obtención del título; todos estos problemas se pueden englobar como algunos factores importantes causantes de un bajo rendimiento académico. (Flores-Ortiz, Rivera-Coronel, \& Sánchez-Cancino, 2016)En los últimos años, ha crecido el interés por conocer los determinantes del rendimiento académico, por lo que se considera importante la necesidad de investigar variables que aporten a la comprensión del hecho del fracaso escolar(López, 2013).

Según la Organización para la Cooperación y el Desarrollo Económico (OCDE), los datos que se tienen de México sobre los estudiantes que ingresan a la universidad son preocupantes, ya que los consideran como dependientes, inseguros, sin resistencia a la frustración, con dificultades para organizar su tiempo, con hábitos de estudio deficientes, con poco tiempo dedicado al estudio durante la semana, sus decisiones se centran en aspectos lúdicos, e ingresan con la idea de que realizar estudios profesionales no requiere gran esfuerzo. (Flores-Ortiz, Rivera-Coronel, \& SánchezCancino, 2016).

Debido a la importancia que adquiere en la educación formal, han surgido numerosas investigaciones dedicadas a esclarecer cuáles son los factores que influyen sobre este rendimiento; por mencionar algunos factores: nivel socioeconómico, escolaridad de los padres, tipo de institución educativa, habilidades de estudio, motivación, etc., no obstante, se ha demostrado que poco es lo que aportan a la planeación e instrumentación de programas preventivos(Caso Niebla \& Hernández Guzmán, 2007)(Zapata, De los Reyes, Lewis, \& Barceló, 2009)

A este respecto, Zapata y cols. (2009) proponen que las variables que inciden en el rendimiento académico se podrían agrupar en: intelectuales/cognitivas, aptitudinales y afectivas. Sin embargo, el estrato socioeconómico, educación de los padres, e incluso, el tamaño de la familia se considera como variables inmodificables.
La neuropsicología cognitiva actual, plantea realizar análisis que particularicen verticalmente cómo las capacidades y procesos cognitivos se relacionan con el aprendizaje, en este sentido, propone analizar no la memoria en general sino mirar qué tipo de memoria es la más relevante en un momento dado del aprendizaje. (Zapata, De los Reyes, Lewis, \& Barceló, 2009) Algunos hallazgos indican que la capacidad de almacenar y procesar material durante periodos cortos de tiempo puede tener un papel crucial en las principales áreas de aprendizaje, ya que, la memoria de trabajo es la responsable de la capacidad de retener y manipular la información (López, 2013).

Así entonces, entender el funcionamiento de la memoria de trabajo y su relación con el aprendizaje académico puede traer grandes beneficios en el ámbito educativo. Como menciona Nieves, Morales y Duarte (2016), las aplicaciones pueden ir desde optimizar significativamente la enseñanza de una determinada área del conocimiento, hasta la detección de problemas de aprendizaje, así como su ayuda (González Nieves, Fernández Morales, \& Duarte, 2016).

En lo que respecta a la carrera de Medicina de la Facultad de Medicina y Nutrición de la UJED (FAMEN) se cuentan con datos acerca de los indicadores sobre la Eficiencia Terminal (ET) así como la reprobación de los alumnos, encontrando que en los últimos 6 años según los anuarios estadísticos de la UJED la ET de los estudiantes ha oscilado entre un 39 a un $50 \%$; por su parte el índice de reprobación se cuenta entre un $40 \%$ a un $45 \%$ en $2017-2018$ siendo estos números motivo para indagar sobre qué es lo que está ocurriendo con los estudiantes que se están rezagando y no concluyen a tiempo con sus estudios, así como, saber de qué manera se puede influenciar el factor cognitivo para tener los porcentajes tan altos de reprobación. (Institucional, Anuario Estadístico, 2015)(UJED, 2017; Institucional, 2016)(Institucional, Anuario Estadístico, 2018) Dada la necesidad del aspecto cognitivo de los alumnos en su rendimiento académico, requiere profundizar en el conocimiento de este constructo, identificando que componente o componentes pueden explicar mejor la relación de la memoria de trabajo y el rendimiento académico. (López, 2013). 
Es imperante la detección a tiempo de factores que influyen en el rendimiento académico de los alumnos, ya que permite a futuro implementar acciones de prevención para mantener y promover su desarrollo estudiantil. (Esquivel Gámez, Gálvez Buenfil, \& BarriosMartínez, 2018).

\section{Rendimiento Académico}

La educación en México y hablando en lo que se refiere a su eficacia y/o eficiencia de las instituciones educativas se ha enfrentado a través de su historia a problemas tales como ET que se deben a diversos factores, tales como económicos, reprobación, programas de estudios obsoletos, factores psicológicos, etc., que hacen que en México el número de estudiantes que ingresan a la educación superior y no logran terminar la carrera están en un promedio del 50\%. (Arriaga Nabor, 2016)

En el caso de la ET tal como lo plantea Toscano de la Torre (2016) esta demuestra los estragos de la reprobación y deserción (rendimiento académico), además que cuando se combina con otros indicadores como la duración de los estudios de egresados y desertores con indicadores de gasto educativo, así vislumbran los gastos adicionales o pérdidas del sistema debido a la inversión en servicios educativos y recursos económicos en estudiantes que no completan. (López Lugo, Villatoro V., MedinaMora, \& Juárez García, 1996) (Enciso Arámbula, 2016)

A nivel gubernamental el gasto nacional en educación creció en un $8.7 \%$ de 2012 a 2017 , pasando de un gasto de 978,436.9 millones de pesos a 1,288,224.6 millones de pesos, esto en proporción al aumento de matrículas escolares, y el gasto promedio durante el ciclo escolar 20162017 por alumno en educación superior fue de 77.5 miles de pesos. Refiriendo lo anteriormente descrito, se traduce que existe un elevado gasto en educación superior por alumno, y esto aumenta con las circunstancias como el bajo rendimiento académico que conlleva a la reprobación generando pérdidas económicas al país.(Estados Unidos Mexicanos, 2017).
El rendimiento académico (RA) ha sido definido como el cumplimiento de las metas, logros u objetivos establecidos en el programa o asignatura que está cursando un alumno en el que intervienen múltiples factores como pueden ser; la edad, el sexo, factores socioeconómicos, la vocación, entre otros; no se conoce un modelo único que describa el rendimiento académico, desde el punto de vista operativo, este indicador se ha limitado a la expresión de una nota cuantitativa.(Batista Rojas, Reyes Delgado, \& Velazquez Medina, 2011) (Santander, 2011) (Quintero, y otros, 2006)

La forma de evaluar el RA está en función de los conocimientos escolares adquiridos previamente y para fines prácticos de esta investigación se toma en cuenta mediante las calificaciones obtenidas, con una valoración cuantitativa; estos resultados permiten la aprobación o reprobación de las materias que componen una curricula. Las notas obtenidas, como un indicador que certifica el logro alcanzado, son un indicador preciso y accesible para valorar dicha situación. (Garbanzo Vargas, 2007)(López Lugo, Villatoro V., Medina-Mora, \& Juárez García, 1996) (González-Olaya, Delgado-Rico, Escobar-Sánchez, \& CárdenasAngelone, 2014)

Como lo menciona Mejía Quintero y Escobar Melo (2012) quienes citan a Cascón (2000): "las calificaciones escolares han sido, son y probablemente seguirán siendo el indicador del nivel educativo adquirido y que a su vez estas son reflejo de las evaluaciones y/o exámenes donde el alumno ha de demostrar sus conocimientos sobre distintas áreas o materias..."

Así también se puede pensar que se tiene una evaluación que refleje por ejemplo, la capacidad para memorizar, o para exponer un tema ante un grupo, e incluso para responder un examen de diferentes modalidades, siempre y cuando cualquiera de éstas refleje los conocimientos transmitidos al alumno. (López Lugo, Villatoro V., Medina-Mora, \& Juárez García, 1996). 
Como en todo proceso se presentan las dificultades entre los estudiantes, unos responden satisfactoriamente mientras que otros tiene dificultades para asimilar los conocimientos que les brinda una institución educativa (Mejía Quintero \& Escobar Melo, 2012); este rendimiento del estudiantado universitario constituye pues un factor imprescindible en el abordaje del tema de la calidad de la educación superior, debido a que es un indicador que permite una aproximación a la realidad educativa. (Garbanzo Vargas, 2007).

Dado que, muchos alumnos en todo el mundo están atrapados en un círculo vicioso de bajo rendimiento que los hace seguir sacando malas notas y perder aún más su compromiso con su escuela, esto tiene consecuencias a largo plazo, tanto para el individuo como para el conjunto de la sociedad y si bien, uno de los objetivos de la OCDE es reducir el número de alumnos con bajo rendimiento, es una manera efectiva de mejorar el rendimiento conjunto de un sistema educativo. (OCDE, 2016).

Durante cada ciclo escolar siempre se ha encontrado que los estudiantes con bajo rendimiento académico son sujetos normales desde el punto de vista intelectual, pero que por diversas causas fallan en sus aprendizajes escolares. La eficiencia diaria en la escuela tal como lo refirió Bricklin (1975) citado en el trabajo de López Lugo (1996) es inferior a lo que podría esperarse de su inteligencia. Podría suponerse que el problema no es por causas ogánicas, y se plantea que es por un uso ineficiente de todas sus capacidades cognitivas y que estas al ser detectadas con claridad, puedan idearse mecanismos que ayuden a prevenir la situación.(López Lugo, Villatoro V., MedinaMora, \& Juárez García, 1996).

Además, la variación individual en el rendimiento académico es ampliamente conocida en cualquier institución educativa y la memoria de trabajo parece ser uno de los componentes cognitivos que están relacionados en estas diferencias individuales. (González Nieves, Fernández Morales, \& Duarte, 2016) Desde esta perspectiva, el rendimiento académico es el resultado de múltiples factores que se cruzan en un momento y en un sujeto particular, no obstante se pretende indagar sobre el factor cognitivo, específicamente hablando de la memoria de trabajo.

\section{Memoria de Trabajo}

La memoria está considerada comúnmente como uno de los atributos más importantes para la vida del ser humano, ya que, es la capacidad que tenemos para almacenar información, acontecimientos pasados y recuperarlos, traer a la conciencia esa información de forma aprendida y gracias a ella podemos saber todo aquello que nos es necesario para poder adaptarnos al medio de una forma óptima. (Portellano Pérez \& García Alba, 2014) (Ostrosky-Solís, Chayo-Dichy, Gómez Pérez, \& Flores Lázaro, 2005)

Los modelos actuales de memoria sugieren 3 tipos de memoria: memoria sensorial, memoria de corto plazo (también conocida como inmediata, primaria o de trabajo) y memoria de largo plazo. La memoria de corto plazo es un almacén de capacidad limitada que codifica información básicamente con características lingüísticas. En general en ella podemos mantener 6 o $7 \pm 2$ ítems o unidades (span), los cuales se olvidan en 6 o 7 segundos, a menos que se repitan continuamente o se manipulen con otras técnicas para que puedan ser transferidos a un almacén más permanente. (Ostrosky-Solís, Chayo-Dichy, Gómez Pérez, \& Flores Lázaro, 2005). Esta memoria a corto plazo se ha subdividido con base a que su funcionamiento no depende de un sistema único, sino de un grupo de sistemas distintos. En tal caso, la memoria de trabajo o memoria operativa según Baddeley y Hitch (1974), citado por Ostrosky y cols. (2005), se resume en otros 3 componentes: el bucle fonológico, un boceto visoespacial y un ejecutivo central, que llevan a cabo procesos de control, manipulación y gestión de la información contenida en la memoria a corto plazo. Posteriormente Baddeley (2000) citado en Portellano (2014) reformuló el modelo y propuso un cuarto elemento, el buffer episódico. En definición la memoria operativa (MO)son "aquellos procesos implicados en el control, regulación, regulación y mantenimiento de información relevante para una determinada tarea al servicio de operaciones cognitivas complejas; también se plantea que es una función de memoria consciente o declarativa con proceso simultaneo con la capacidad funcional para mantener y procesar información al mismo tiempo (Escudero Cabarcas \& Pineda Alhucema, 2017). 
Así, esta memoria empezó a considerarse como una entidad que no sólo almacenaba información, sino que tenía un papel más activo y dinámico(Portellano Pérez \& García Alba, 2014). Implica el procesamiento y recuperación de la información de manera inmediata bajo una estructura que permite la adquisición y procesamiento de la información (proveniente del medio físico) y que lleva al reconocimiento de los elementos del medio, que al ingresar a través de los órganos de los sentidos, dan repuesta a otros procesos de orden superior, se basa en gran medida, mas no exclusivamente, en los lóbulos frontales(Escudero Cabarcas \& Pineda Alhucema, 2017).

El bucle fonológico es el componente responsable de preservar la información basada en el lenguaje, el bucle tendría por misión almacenar información de tipo lingüístico (López-Magdalena, 2011). A su vez consta de dos subcomponentes: un almacén fonológico y un proceso de control articulatorio. El primero retiene información fonológica y linguística (como retención de dígitos), mientras que el segundo está relacionado con el habla subvocal (por ejemplo, para tareas de aprender un número de teléfono o como aprender a leer, lectura de comprensión o adquirir nuevo vocabulario).(Baddeley, Working Memory: The Interface between Memory and Cognition, 1992).

La recuperación y la re-articulación se utilizan para actualizar las huellas de memoria, y el espacio de la memoria de trabajo está limitado por la cantidad de material que se puede articular antes de que el primer elemento se desvanezca. La longitud de las palabras y la similitud entre los elementos influyen fuertemente en el rendimiento en las pruebas de memoria de trabajo verbal.(Baddeley, Working memory: looking back and looking forward, 2003).

El segundo componente es la agenda visoespacial, es el sistema responsable de preservar y procesar información de naturaleza visual y espacial proveniente tanto del sistema de percepción visual como del interior de la propia mente.
Este sistema cumple con la generación y manipulación de imágenes mentales, de modo que las interferencias espaciales que no requieren procesamiento visual llevan a una disminución del rendimiento de tareas de seguimiento de secuencias en movimiento, pero no en el recuerdo de imágenes estáticas o colores.(Castro \& Valeska, 2017).

Según Magdalena López (2013) quien cita a Baddeley (2003) parece que este sistema estaría involucrado en tareas de lectura, participando en el mantenimiento de una representación de la página y su diseño y de que permanezcan estables facilitando tareas como el movimiento de los ojos con precisión desde el final de una línea a principios de la siguiente. Igualmente plantea que la agenda podría tener un papel en la adquisición de conocimiento semántico acerca de la apariencia de los objetos y cómo usarlos y en la comprensión de los sistemas complejos tales como maquinarias, así como para la orientación espacial y los conocimientos geográficos. (Magdalena, 2013).

Según Baddeley (2003) es necesario especificar los procesos que se le atribuyen a este sistema de activación de supervisión y explicarlos de forma clara. Los procesos necesarios son enfocar, dividir y cambiar la atención, y conectar la memoria de trabajo con la memoria a largo plazo. (Baddeley, Working memory: looking back and looking forward, 2003). Para explicar esta capacidad, se tiene un tercer buffer, este integra a los dos anteriores con la información de la memoria a largo plazo, denominado como buffer episódico. Baddeley lo interpreta como un sistema multifuncional que opera como almacén auxiliar en caso de sobrecargarse los buffers principales; además parece tener la función de almacenar información compleja (como dimensión temporal). (Portellano Pérez \& García Alba, 2014). Los tres componentes descritos anteriormente, están controlados por un sistema atencional de capacidad limitada, llamado "el ejecutivo central", que maneja la información almacenada en los otros dos sistemas, y a su vez, se divide en tres diferentes subprocesos: inhibición, cambio y actualización. 
Este determina cuándo se almacena la información en los diferentes buffers, es decir, integra y coordina la información y proporciona el modo por el cual la información se puede inspeccionar, transformar y manipular a nivel cognitivo.(González Nieves, Fernández Morales, \& Duarte, 2016). Este es el componente más complejo de la memoria de trabajo dado que lleva a cabo el control voluntario y toma de decisiones, estando estrechamente relacionado con la atención y la experiencia consiente. Neurofisiológicamente es asociado con el córtex prefrontal dorso lateral (CPDL) y medial y con regiones parietales de la corteza cerebral. (Escudero Cabarcas \& Pineda Alhucema, 2017).

De hecho, las funciones del ejecutivo central podemos considerarlas propiamente como funciones ejecutivas, estrechamente relacionadas con estructuras cerebrales como la corteza prefrontal izquierda.(González Nieves, Fernández Morales, \& Duarte, 2016).

El rendimiento en tareas de memoria depende pues de la habilidad del individuo para manipular unidades pequeñas de información. Las implicaciones para la práctica son (Etchepareborda \& Abad-Mas, 2005):

- La conveniencia de que la información a manipular por el individuo sea lo suficientemente comprensible como para que pueda identificar los elementos que la componen y organizarla de acuerdo con sus esquemas.

- Entrenamiento o práctica que permite ampliar los límites de espacio y tiempo que tiene nuestra MT.

- Problemas que pueden acarrear, para el aprendizaje, estímulos interferentes o distractores que impidan aprender.

- La organización de la información facilitará su recuperación.

- La información puede ser organizada jerárquicamente, por orden alfabético, por categorías, por número de elementos, etc. Por tanto, la incorporación de un nuevo dato, puede dar lugar la reorganización o modificación de su estructura.

\section{Evaluar la memoria operativa (Portellano)}

Se ha reconocido que la $\mathrm{MO}$ es limitada en su capacidad, lo que la configura como posible fuente de diferencias entre individuos bajo demanda cognitiva. Este hecho podría explicar el nivel de desempeño variables en comprensión lectora, razonamiento y rendimiento académico. (Esquivel Gámez, Gálvez Buenfil, \& BarriosMartínez, 2018). Existen diversas modalidades en las pruebas de memoria de trabajo, la esencia de cada una de ellas es que los sujetos tienen que mantener una información de cantidad limitada por un tiempo relativamente corto, mientras se realiza una acción basada en esta información. (Flores Lázaro, 2006).

De forma constante, en nuestra actividad diaria, estamos sometidos a actividades mentales en las que se pone en juego la memoria operativa. Dependiendo de su ejecución, esas tareas se llevarán a cabo de una forma óptima o no. Cuando nos dan un número de teléfono y no tenemos un cuaderno en el que apuntar, somos conscientes de que podemos olvidarlo, si no todos los números, sí alguno. Si nos dirigimos a alguna dirección y no sabemos cómo ir, podemos preguntar cómo se va, las indicaciones que nos dan se convertirán en representaciones mentales que debemos mantener y manipular mentalmente para no perderlas.(Portellano Pérez \& García Alba, 2014).

Una de las divisiones importantes ha sido la división verbal/no verbal. En la que se ha encontrado que la CPF dorsolateral izquierda se relaciona más con las pruebas verbales y la $\mathrm{CPF}$ dorsolateral derecha se relaciona más con las pruebas viso-espaciales. Aunque se ha podido identificar que el procesamiento de la identidad de los objetos (¿qué?) se encuentra soportado por las porciones ventrales de la CPF, mientras que su posición espacial (¿dónde?) por las porciones dorsolaterales de la CPF derecha. (Flores Lázaro, 2006). Ahora bien, para valorar al bucle fonológico este se puede valorar mediante tareas que impliquen la repetición de series silábicas ascendentes. Estas series pueden ser dígitos, palabras o pseudopalabras. Tareas como realizar una serie de varias operaciones aritméticas básicas $(3 \times 4+8) \mathrm{y}$, al finalizar, tratar de memorizar una palabra que acompaña a cada operación, pueden labores estándar para la valoración de la MO.(Portellano Pérez \& García Alba, 2014). 
Para el estudio del buffer visoespacial se han utilizado habitualmente dos tipos de materiales: visual (colores, formas, caras, imágenes, etc.) y espacial (localizaciones). Existe una distinción fundamental entre la información visual y la espacial: el primero ("visual cache") provee un almacén temporal para la información relativa al color y la forma; el segundo se ocupa tanto de la manipulación de la información de secuencias de movimientos, como del repaso activo de esa información visual en MT. Como mencionan Darling, Della Sala y Logie (2007) citados por Mamarella y colaboradores (2014), los primeros diferencian entre un proceso pasivo y uno activo en función del tipo de información: a) pasivo, de conservación de los estímulos (naturaleza visual), y b) activo, caracterizado por una mayor elaboración y procesamiento de los estímulos (de carácter espacial). (Pérez, Mammarella, Del Prete, Bajo, \& Cornoldi, 2014).

Valorar el ejecutivo central es más complejo dada la implicación de funciones ejecutivas y que siempre se verán implicados algunos de los componentes esclavos de la memoria operativa. Tareas que pueden acercarse para valorarlo pueden ser tareas de tipo repetición de dígitos inversos y/o dígitos que se presentan al sujeto de forma desordenada y que debe ordenarlos al repetirlos(Portellano Pérez \& García Alba, 2014).

\section{Pregunta de Investigación}

¿Cuál es la relación entre la memoria de trabajo con el rendimiento académico de los estudiantes de Medicina de la FAMEN UJED?

\section{Hipótesis}

Existe una relación entre la memoria de trabajo con el rendimiento académico de los estudiantes de Medicina de la FAMEN UJED

\section{Objetivo General}

Determinar la relación entre la memoria de trabajo con el rendimiento académico de los estudiantes de Medicina de la FAMEN UJED

\section{Objetivos Específicos}

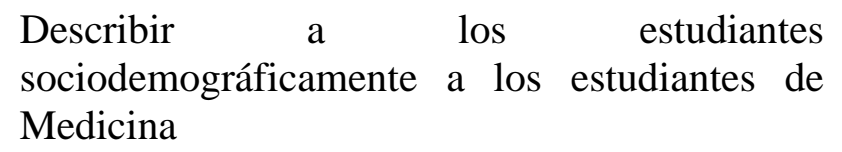

Describir los puntajes evaluados de la memoria de trabajo mediante la Batería Neuropsicológica Breve en español (NEUROPSI).

Identificar el rendimiento académico de los estudiantes mediante las calificaciones que registraban hasta el momento del estudio. de interés.

Examinar la relación entre las variables

\section{Materiales y Métodos}

Tipo de Estudio: se realizará un estudio transversal-observacional, de tipo descriptivocorrelacional

Diseño de investigación: no experimental, retrospectivo-retrolectivo con una muestra no probabilística por conveniencia de estudiantes de los semestres correspondientes de $2^{\circ}$ a $10^{\circ}$ semestre de la carrera de Medicina con previa firma de consentimiento informado.

Criterios de Inclusión, Exclusión y Eliminación: Los criterios para la inclusión al estudio son alumnos sanos en edades comprendidas entre los 18-25 años, con registros de expediente completos, con firma de consentimiento informado. Se excluyeron aquellos participantes en los que se haya identificado alguna enfermedad o patología neurológica o psiquiátrica. Se eliminaron aquellos expedientes que se encontraran con información incompleta.

Universo de estudio: El universo de estudio constaba de un total de 352 registros. Atendiendo los criterios de inclusión y exclusión se tuvo un total de 270 registros completos.

\section{Procedimiento}

Para el desarrollo de este trabajo se partió de una revisión teórica de investigaciones anteriormente realizadas, para lograr tener una aproximación conceptual satisfactoria, y entender en forma correcta las variables de estudio. Se seleccionó la muestra identificando los registros completos que se tenían de los estudiantes de la carrera de Medicina de $2^{\circ}$ a $10^{\circ}$ semestre quienes cumplieron con los criterios para participar en el estudio. 
Hoy en día no existe algún instrumento científicamente válido para medir el rendimiento académico, como en otros constructos sociales. Tomando en cuenta lo que se reporta en la bibliografía se toma en cuenta al RA como la expresión de una nota cuantitativa (escala del 6 al 10) obteniendo el promedio general con el que los estudiantes contaban al momento del estudio. Se realizó la aplicación de una encuesta clínica para conocer justamente los antecedentes patológicos y no patológicos de los participantes. Posteriormente se hizo la aplicación de la Batería Neuropsicológica Breve en español (NEUROPSI) de manera individual, la cual tiene una duración aproximada de 20 a 25 minutos en población sin alteración cognitiva, en el Instituto de Investigación Científica (IIC) por profesionales en Comunicación Humana capacitados y entrenados en el empleo de la Batería Neuropsicológica. Tomando en cuenta la escolaridad del sujeto se puede clasificar la ejecución en: normal, alteración leve o limítrofe, alteración moderada y alteración severa.

La prueba elegida del NEUROPSI para evaluar el bucle fonológico fue la tarea de Memoria Verbal Espontánea donde se le pide al sujeto enunciar una lista de 6 palabras que el examinador proporciona en tres ensayos consecutivos y obtener el número promedio de palabras repetidas. Esta prueba requiere principalmente almacenamiento de información auditiva a corto plazo en la memoria de trabajo. (López, 2013)(Portellano Pérez \& García Alba, 2014). Para la evaluación de la agenda visoespacial se utilizó el Test de Copia de Figura Semicompleja, esta técnica evalúa habilidades visomotoras, percepción visual y memoria visual inmediata, además de valorar habilidades de planificación y organización. El uso de la memoria no verbal se involucra en la organización y reproducción de diseños complejos (López, 2013).

La prueba elegida para evaluar el componente Ejecutivo Central fue el test de Dígitos en Regresión o Inversos, aquí se deben repetir series de números que van desde dos hasta ocho dígitos, en orden inverso (o contrario) al presentado por el examinador. Esta prueba evalúa la habilidad para mantener una cifra en la memoria, al mismo tiempo que se la reordena. Implica la manipulación activa de la información almacenada, poniendo en funcionamiento el control ejecutivo de la memoria de trabajo (López, 2013).
La recolección y captura de la información se registró en el programa Excel para su análisis estadístico. En primer lugar, se realizó una prueba de bondad de ajuste para conocer la normalidad de los datos con el cual se estableció el uso del coeficiente de correlación de Spearman para cada una de las medidas de la memoria de trabajo y el rendimiento académico, se reportarán las medidas de tendencia central (media, mediana y moda) y de dispersión para los puntajes cuantitativos tanto de la evaluación de la memoria como del rendimiento; en el caso de las variables cualitativas se usarán medidas de frecuencias y proporciones (como sexo y semestre).

\section{Resultados}

A continuación, se presentan los estadísticos descriptivos del estudio. La muestra estuvo conformada por 270 participantes que contaron con todos los registros completos, 118 (43.7\%) participantes del género masculino 152 (56.3\%) del género femenino), todos ellos pertenecientes a los distintos semestres $\left(2^{\circ}\right.$ a $\left.10^{\circ}\right)$. La edad de los participantes oscilo entre los 18 y 25 , la edad promedio es de 21.47 años \pm 0.98 , con una mediana de 21 y moda de 20 . Se realizó una diferenciación entre aquellos alumnos que tuvieran un estatus académico regular (es decir, aquellos alumnos que no hubieran recursado alguna materia) y estatus no regular (aquellos alumnos que hubieran recursado al menos 1 materia), teniendo que el $53.3 \%$ (144) de la muestra estudiada son alumnos que tienen un estatus no regular frente a un 46.7 (126) que no han recursado alguna materia.

En cuanto al RA tomado en cuenta como un valor cuantitativo del promedio de las calificaciones de los alumnos tiene una media de $8.47 \pm 0.25$, mediana y moda de 8.5 , el valor mínimo de 7.4 y el máximo de 9.7 .

En la tabla 1 se muestran los resultados descriptivos de la prueba de memoria verbal espontánea con la que se evaluó el bucle fonológico. Según los puntajes de la batería Neuropsicológica un $96.6 \%$ de los participantes se encuentran dentro de los valores normales. 


\begin{tabular}{|c|c|c|}
\hline Puntaje* & Frecuencia & Porcentaje \\
\hline 2 & 1 & .4 \\
\hline 3 & 8 & 3 \\
\hline 4 & 50 & 18.5 \\
\hline 5 & 164 & 60.7 \\
\hline 6 & 47 & 17.4 \\
\hline Total & 270 & 100 \\
\hline \multicolumn{3}{|c|}{$\begin{array}{l}\text { *Puntajes: Alteración severa }\left(\begin{array}{lll}0 & \text { a } & 2\end{array}\right) \text {, Alteración } \\
\text { moderada (3), Normal (4 a 6) }\end{array}$} \\
\hline
\end{tabular}

Tabla 1 Puntajes de Memoria Verbal Espontánea

En la tabla 2 se muestran los resultados para la evaluación de la agenda visoespacial mediante la figura semicompleja. Aquí se observa que un $22.6 \%$ de los alumnos presentaron una alteración entre moderada y severa y el resto tienen puntajes dentro de valores normales.

\begin{tabular}{|c|c|c|}
\hline Puntaje* & Frecuencia & Porcentaje \\
\hline 6 & 1 & .4 \\
\hline 9 & 1 & .4 \\
\hline 10 & 4 & 1.5 \\
\hline 10.5 & 2 & .7 \\
\hline 11 & 53 & 19.6 \\
\hline 11.5 & 4 & 1.5 \\
\hline 12 & 205 & 75.9 \\
\hline Total & 270 & 100 \\
\hline \multicolumn{3}{|c|}{$\begin{array}{l}\text { *Puntajes: Alteración severa (0 a 10.5), Alteración } \\
\text { moderada (11), Normal (11.5 a 12) }\end{array}$} \\
\hline
\end{tabular}

Tabla 2 Puntajes de la Copia de Figura Semicompleja

Por su parte en la Tabla 3 se observan los resultados de la evaluación para el componente ejecutivo central. Sólo un $7.4 \%$ de la muestra tuvo alteración moderada para la realización de la prueba, el resto se encuentra dentro de valores normales.

\begin{tabular}{|c|c|c|}
\hline Puntaje* & Frecuencia & Porcentaje \\
\hline 2 & 20 & 7.4 \\
\hline 3 & 63 & 23.3 \\
\hline 4 & 95 & 35.2 \\
\hline 5 & 59 & 21.9 \\
\hline 6 & 33 & 12.2 \\
\hline Total & 270 & 100 \\
\hline \multicolumn{3}{|c|}{$\begin{array}{l}\text { *Puntajes: Alteración severa (0-1), Alteración } \\
\text { Moderada (2), Normal (3-5), Normal alto (6) }\end{array}$} \\
\hline
\end{tabular}

Tabla 3 Puntajes de Dígitos en Regresión

Para el análisis de las variables de interés se realizó una correlación de Spearman, ya que al hacer una bondad de ajuste mediante Kolmogorov-Smirnov resultaron no normales todas las variables. En la tabla 4 se presentan los resultados del análisis de correlación aplicado entre el RA y las medidas de MT.
Lo que se obtuvo es que en ninguna de las mediciones de la MT se encontró correlación frente al rendimiento académico de los participantes.

\begin{tabular}{|c|c|c|c|}
\hline & \multicolumn{3}{|c|}{ Memoria de trabajo } \\
\hline & $\begin{array}{l}\text { Dígitos en } \\
\text { Regresión }\end{array}$ & $\begin{array}{c}\text { Memoria } \\
\text { Verbal } \\
\text { Espontánea }\end{array}$ & $\begin{array}{c}\text { Copia de } \\
\text { Figura } \\
\text { semicompleja }\end{array}$ \\
\hline $\mathrm{r}^{*}$ & 0.02 & 0.05 & 0.08 \\
\hline $\mathrm{p}$ & 0.68 & 0.39 & 0.14 \\
\hline
\end{tabular}

Tabla 4 Relación entre Rendimiento Académico (RA) y medidas de memoria de trabajo (MT)

A pesar de no ser el objetivo principal, se realizó un segundo análisis estadístico mediante comparación de medias. Se dividió a los estudiantes en dos grupos, aquellos ubicados como estatus regular y los de estatus no regular tomando en cuenta el recusamiento o no de una o más materias y estudiantes con bajo y alto rendimiento académico.

Para determinar a los estudiantes con un rendimiento académico bajo o con rendimiento alto se tomó en cuenta el promedio de las calificaciones del total de la muestra; aquellos quienes estuvieran por debajo de la media son considerados con rendimiento académico bajo, mientras que aquellos alumnos que estuvieran por encima de la media se consideran como el grupo con rendimiento académico alto.

Dentro del primer grupo (bajo rendimiento) se encuentran 104 alumnos del total de la muestra de los cuales, el $65.4 \%$ son regulares y el $36.4 \%$ son no regulares, es decir, la mayoría de los estudiantes que están dentro de este grupo, han tenido una trayectoria escolar favorable, sin haber recursado alguna materia. Además, las características de las habilidades de la memoria de trabajo se encuentran dentro de los rangos normales marcados por la batería neuropsicológica breve en español.

En el segundo grupo señalado como alto rendimiento académico hay 148 estudiantes, de los cuales el $31.1 \%$ son estudiantes regulares y el $68.9 \%$ son no regulares, esto se puede traducir en que, los alumnos que han recursado alguna materia y se han rezagado en su trayectoria escolar, luego de llevar por segunda o tercera ocasión alguna materia consiguen mejores calificaciones que aquellos quienes son regulares. 
Al igual que el grupo anterior cuenta con que la memoria de trabajo en sus tres componentes los alumnos están dentro del rango de normalidad marcado por la prueba. Para evaluar la existencia de diferencias entre grupos, se realizó un análisis mediante la prueba $U$ de Mann-Whitney. Los resultados no indican alguna diferencia estadísticamente significativa entre las subpruebas para la evaluación de los componentes de la Memoria de Trabajo, no obstante, si se encontró una diferencia estadísticamente significativa $(\mathrm{p}=.000)$ entre el rendimiento académico los estudiantes regulares y los no regulares, siendo mejores las calificaciones en este último grupo.

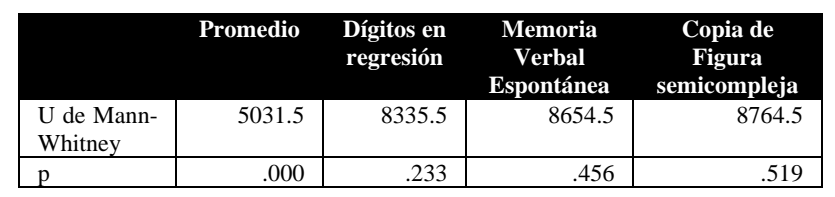

Tabla 5 Comparación entre Estatus Escolar (Regular y no Regular)

\section{Agradecimiento}

Al Instituto de Investigación Científica de la Universidad Juárez del Estado de Durango (UJED) y a la Facultad de Medicina y Nutrición (FAMEN) de la UJED por haber el apoyo invaluable para la realización del proyecto.

\section{Discusión y Conclusiones}

Entender qué es la Memoria de Trabajo, cómo funciona y cómo está relacionada con el aprendizaje es muy importante para la educación en cualquier edad, ya que este conocimiento brinda el potencial para entender las experiencias que se suscitan en aula, así como para aplicar adecuadamente intervenciones en caso de ser necesario.

Dado que las demandas más comunes en las que se ve involucrada este tipo de memoria son: seguir instrucciones, demandas simultáneas de procesamiento, seguir el proceso de tareas complejas y recordar distintos episodios; dichos requerimientos son necesarios para la mayoría de procesos mentales dentro del salón de clase. (González Nieves, Fernández Morales, \& Duarte, 2016)
Además, es bien sabido que en todo proceso educativo existen alumnos que responden satisfactoriamente mientras que otros presentan mayores dificultades, a pesar de ser sujetos considerados como sanos desde el punto de vista intelectual y no obstante fallan en sus aprendizajes escolares. (Mejía Quintero \& Escobar Melo, 2012) (López Lugo, Villatoro V., Medina-Mora, \& Juárez García, 1996).

Estas diferencias han sido estudiadas en diversas poblaciones de estudiantes, y se ha encontrado que existe una relación predictiva entre presentar dificultades en la memoria de trabajo y un bajo rendimiento académicoeducativo y según Alloway, Banner y Smith (2010) se asocian con bajos resultados de aprendizaje y son estudiantes que de manera frecuente quedan rezagados respecto a sus compañeros.(Alloway, G.E., \& Smith, 2010).

Dicho lo anterior, dentro de los resultados, se observan que poco más del $50 \%$ de los alumnos han recursado una o más materias por motivos de reprobación luego de un ciclo académico (un semestre), a pesar de ser sujetos clínicamente sanos; siguiendo a Zapata y colaboradores (2009) mencionan que la neuropsicología cognitiva propone particularizar la investigación de los distintos procesos cognitivos y cómo estos se relacionan con el aprendizaje, lo cual es un punto de observación para profundizar la investigación en esta población y reconocer qué otras habilidades neurocognitivas pueden ser factores determinantes para tener un rendimiento académico satisfactorio.

Respecto a los resultados de la correlación de las variables de interés, no indican la existencia de alguna relación entre ambas mediciones, rechazando la hipótesis planteada en un principio, y concordando en este caso con los resultados que obtuvieron Zapata y colaboradores (2009) en su estudio en estudiantes colombianos. Al realizar el segundo análisis mediante la comparación únicamente se obtuvo una diferencia en cuanto a las calificaciones de los grupos regular y no regular, no así para los componentes de la memoria de trabajo. 
Estos resultados sugieren que, las habilidades y recursos que emplean los estudiantes en cuanto a su memoria de trabajo son similares, no así, el rendimiento académico expresado mediante sus calificaciones adquiere un impacto debido a que son los estudiantes no regulares quienes tienen mejores calificaciones en comparación con quienes no han reprobado.

Ahora bien, un estudiante que ha recursado una o más de una materia impacta en su trayectoria escolar, en los indicadores escolares y de manera estadísticamente significativa en su rendimiento académico, tal como los resultados lo muestran en este estudio, y que esto sea un impulso, que se propongan mejorar o encuentren motivación para esforzarse en su proceso de enseñanza-aprendizaje (FloresOrtiz, Rivera-Coronel, \& Sánchez-Cancino, 2016) y conseguir mejorar sus calificaciones, a pesar de no haber diferencias en las habilidades de la memoria, así pues ¿Qué variables o dimensiones de naturaleza personal, determinan el rendimiento académico de estudiantes de la licenciatura en Medicina?

Dado que el rendimiento académico es resultado de múltiples factores (en este caso se particularizó en factores cognitivos), es difícil de medir y que en esta población se ha encontrado poca bibliografía que describan las variables cognitivas, sin embargo, mantenemos la idea que planteaba López Lugo y colaboradores (1996), en este caso de aquellos estudiantes que no son regulares, que cuentan con un bajo rendimiento académico y con ciertas dificultades en la MT, se sugiere que es debido a que tienen un uso ineficiente de sus capacidades cognitivas que no les permiten desempeñarse satisfactoriamente.

Tal como lo afirman Zapata y colaboradores (2009) no en todos los casos, una buena o mala memoria de trabajo se refleja con un alto o bajo rendimiento académico, en este estudio se encontró en las distintas subpruebas que existen casos con alguna alteración (moderada o severa) marcados por la batería neuropsicológica, no obstante, a pesar de tener un rendimiento bajo en MT algunos participantes cuentan con calificaciones consideradas como altas y viceversa, hay participantes con bajo rendimiento académico y dificultades en el proceso de MT, que suponen una repercusión en los procesos de aprendizaje y que además afectan su trayectoria académica.
Además, la educación en México enfrenta dificultades en el tema de sus indicadores (eficiencia terminal, reprobación, deserción, etc.) que son reflejo del rendimiento de los estudiantes de cualquier institución y que requieren soluciones e intervenciones eficaces, dado que se vislumbran gastos adicionales o pérdidas del sistema de cada alumno que cursa una carrera universitaria, se busca economizar los recursos y sean mejor aprovechados. (López Lugo, Villatoro V., Medina-Mora, \& Juárez García, 1996) (Enciso Arámbula, 2016)

Dentro de las fortalezas de esta investigación se encuentra que la muestra seleccionada, está marcada como una población en la que poco se ha hecho en investigación en el contexto educativo en México, no obstante, se han encontrado trabajos similares mayormente son realizados en población infantil y hechas en población hispano hablante (Fonseca Estupiñon, Rodríguez Barreto, \& Parra Pulido, 2016) (Zapata, De los Reyes, Lewis, \& Barceló, 2009).

Así mismo, se recomienda para futuras investigaciones el uso de otros instrumentos para la evaluación de la memoria de trabajo, y para el rendimiento académico el uso de otros indicadores como: horas de clase recibidas, número de créditos, horas de estudios, horas extracurriculares, que den un mayor sustento objetivo de este rendimiento dado que no hay un instrumento actualmente que de un parámetro de ello.

Igualmente se sugiere analizar y/o comparar el fenómeno de estudio con otras carreras e indagar de qué forma se puede comportar las variables de estudio.

Tradicionalmente se piensa que el uso efectivo de los recursos cognitivos se refleja en un aprendizaje sólido y buenas calificaciones; en conclusión, a pesar que los estudiantes presenten dificultades en algún componente o tengan un adecuado funcionamiento de la memoria de trabajo no determina o garantiza un alto o bajo rendimiento académico. 
Se concluye que el rendimiento académico es resultado de múltiples factores que se interrelacionan e intervienen en un momento dado y de manera individual en cada persona siendo difícil de traducir a un elemento en concreto como se ha venido haciendo, expresándose en notas y promedios como un elemento objetivo, ya que inciden variables subjetivas: afectivas, motivacionales, de personalidad, aptitudinales, entre otras y cada una de ellas puede tener componentes propios que hacen aún más difícil la tarea de análisis de este rendimiento.

Finalmente, se propone establecer esquemas de atención para aquellos alumnos que presentan dificultades en cuanto a memoria de trabajo, aquellos que tengan un bajo rendimiento académico, que hayan recursado materias dado que se encuentran en riesgo de ser dados de baja y pueden provocar pérdidas económicas y del sistema, puesto que representan una vía apropiada de intervención. De tal forma, que la intervención logre el desarrollo de mejores habilidades se optimicen los conocimientos, que sea una opción de ayuda para los estudiantes tal como lo forman las tutorías, orientación pedagógica o psicoeducativa, que favorezca la consolidación de factores que inciden en el rendimiento académico y beneficiar a la población que presenta problemas escolares al igual que a quienes no presenten problemas específicos.

\section{Referencias}

Alloway, T., G.E., B., \& Smith, P. (2010). Working memory, and cognitive styles in adolescents' attainment. British Journal of Educational Psychology, 567-581.

Arriaga Nabor, O. M. (2016). La deserción estudiantil en la educación de nivel superior: estudio de caso Universidad Autónoma de Nayarit . En R. Enciso Arámbula, La Universidad y sus Estrategias de Vinculación (págs. 9-11). Nayarit: UTP.

Baddeley, A. (1992). Working Memory: The Interface between Memory and Cognition. Journal of Cognitive Neuroscience, 281-288.

Baddeley, A. (2003). Working memory: looking back and looking forward. Nature Reviews Neuroscience, 829-839.
Baddeley, A. (2012). Working Memory: Theories, Models, and Controversies. Anual Reviews Psychology, 1-29.

Batista Rojas, O., Reyes Delgado, R. Á., \& Velazquez Medina, L. E. (2011). Factores relacionados con el rendimiento académico en las asignaturas Clínica I y II. EduMecentro, 8391.

Caso Niebla, J., \& Hernández Guzmán, L. (2007). Variables que inciden en el rendimiento académico de adolescentes mexicanos. Revista Latinoamericana de Psicología, 487-501.

Castro, D., \& Valeska, A. (2017). Contribución de los Componentes de la Memoria de Trabajo a la Eficiencia en Aritmética Básica Durante la Edad Escolar. Psykhe, 1-17.

Enciso Arámbula, R. (2016). La Universidad y sus Estrategias de Vinculación. Tepic, Nayarit: Universidad Tecnocientífica del Pacifico S.C.

Escudero Cabarcas, J. M., \& Pineda Alhucema, W. F. (2017). Memoria de Trabajo: El modelo multicomponente de Baddeley, otros modelos y su rol en la práctica clínica. En M. J. Bahamón, \& e. al., Estudios actuales en Psicología. Perspectivas en clínica y salud (págs. 13-41). Colombia: Mejoras.

Esquivel Gámez, I., Gálvez Buenfil, K. E., \& Barrios-Martínez, F. L. (2018). Memoria Operativa: desarrollo, evaluación y aplicación de pruebas en línea para su medición. Innovación, Tecnología y Liderazgo en los entornos educativos, 180-193.

Estados Unidos Mexicanos, G. d. (Agosto de 2017). 5to Informe de Gobierno. Ciudad de México, México.

Etchepareborda, M., \& Abad-Mas, L. (2005). Memoria de trabajo en los procesos básicos del aprendizaje. Rev Neurol, 79-83.

Flores Lázaro, J. C. (2006). Neuropsicología de Lóbulos Frontales. Villahermosa, Tabasco: Universidad Juárez Autónoma de Tabasco. 
Flores-Ortiz, M. E., Rivera-Coronel, H., \& Sánchez-Cancino, F. (2016). Bajo Rendimiento Académico: Más allá de los factores sociopsicopedagógicos. Revista Digital Internacional de Psciología y Ciencia Social, 2(1), 95-104. Obtenido de http://dx.doi.org/10.22402/j.rdipycs.unam.2.1.2 016.60.95-104

Fonseca Estupiñon, G. P., Rodríguez Barreto, L. C., \& Parra Pulido, J. H. (2016). Relación entre funciones ejecutivas y rendimiento académico por asignaturas en escolares de 6 a 12 años. Hacia promoc. salud., 41-58. doi:DOI: 10.17151/hpsal.2016.21.2.4

Garbanzo Vargas, G. M. (2007). Factores asocIados al rendImIento académIco en estudIantes unIversItarIos, una reFlexIón desde la calIdad de la educacIón superIor pública . Revista Educación, 43-63.

González Nieves, S., Fernández Morales, F. H., \& Duarte, J. E. (2 de Julio-Diciembre de 2016). Memoria de trabajo y aprendizaje: Implicaciones para la Educación. Saber, Ciencia y Libertad, 11(2), 147-162.

González-Olaya, H. L., Delgado-Rico, H. D., Escobar-Sánchez, M., \& Cárdenas-Angelone, M. E. (2014). Asociación entre el estrés, el riesgo de depresión y el rendimiento académico en estudiantes de los. FEM: Revista de la Fundación Educación Mëdica, 17(1), 47-54.

Institucional, U. D. (2015). Anuario Estadístico. Durango, Durango, México. Recuperado el 06 de 02 de 2019, de https://www.ujed.mx/doc/publicaciones/anuario s-estadisticos/Anuario_estadistico_2015.pdf

Institucional, U. D. (2016). Anuario Estadístico. Durango, Durango, México. Recuperado el 06 de 02 de 2019, de https://www.ujed.mx/doc/publicaciones/anuario s-estadisticos/Anuario_estadistico_2016.pdf

Institucional, U. D. (2018). Anuario Estadístico. Durango, Durango, México. Recuperado el 06 de 02 de 2019, de https://www.ujed.mx/doc/publicaciones/anuario s-estadisticos/Anuario_estadistico_2018.pdf
López Lugo, E. K., Villatoro V., J., MedinaMora, E., \& Juárez García, F. (1996). Autopercepción del Rendimiento Académico en Estudiantes Mexicanos. Revista Mexicana de Psicología, 37-47.

López, M. (30 de Septiembre de 2013). Rendimiento Académico: $\mathrm{Su}$ relación con la memoria de trabajo. Revista Actualidades Investigativas en Educación, 13(3), 1-19. Obtenido de http://www.redalyc.org/articulo.oa?id=4472987 8008

López-Magdalena. (2011). Memoria de trabajo y Aprendizaje: Aportes de la Neuropsicología. Cuad. Neuropsicol., 25-47.

Magdalena, L. (2013). Diferencias en el desempeño de la memoria de trabajo: un estudio en niños de diferentes grupos sociales. Revista nacional e internacional de educación inclusiva, 109-119.

Mejía Quintero, E., \& Escobar Melo, H. (2012). Caracterización de procesos cognitivos de memoria, lenguaje y pensamiento, en estudiantes con bajo y alto rendimiento académico. Perspect. Psicol., 123-138.

OCDE. (2016). Estudiantes de bajo rendimiento. Por qué se quedan atrás y cómo ayudarles a tener éxito. Unión Europea: OCDE.

Ostrosky-Solís, F., Chayo-Dichy, R., Gómez Pérez, E., \& Flores Lázaro, J. C. (2005). ¿Problemas de Memoria? Un programa para su estimulación y rehabilitación. Edo. de México: LP.

Pérez, A., Mammarella, I., Del Prete, F., Bajo, T., \& Cornoldi, C. (2014). Capacidad geométrica y memoria visoespacial en población adulta. Psicología, 225-249.

Portellano Pérez, J. A., \& García Alba, J. (2014). Neuropsicología de la atención, las funciones ejecutivas y la memoria. Vallehermoso, Madrid: Síntesis.

Quintero, S. B., Ortiz, M. D., Alvira, C. E., Acosta, A. M., Castaño, S. A., Álvarez, A. R., \& González, S. P. (2006). Evaluación de la relacíon entre rendimiento académico y estrés en estudiantes de Medicina. Medunab, 9(3), 198205. 
Santander, O. A. (2011). El rendimiento académico, un fenómeno de múltiples relaciones y complejidades. Revista Vanguardia Psicológica clínica teórica y práctica, 2(2), 144173.

UJED, D. d. (2017). Anuario Estadístico. Anuario Estadístico UJED. Durango, Durango, México. Recuperado el 06 de 02 de 2019, de https://www.ujed.mx/doc/publicaciones/anuario s-estadisticos/Anuario_estadistico_2017.pdf

Zapata, L. F., De los Reyes, C., Lewis, S., \& Barceló, E. (2009). Memoria de trabajo y rendimiento académico en estudiantes de primer semestre de una universidad de la ciudad de barranquilla. Psicología desde el Caribe, 66-82. 